\title{
A Molecular Dynamics Study on the Miscibility and Morphology of Polyester Blends used in Coil Coatings
}

Matthew Wearon *'Brendan Howlin¹, Chris Lowe², Marie-Laure Abel, John F Watts

*m.wearon@surrey.ac.uk Department of Mechanical Engineering Sciences,

Faculty of Engineering and Physical Sciences, University of Surrey, Guildford, GU2 7XH, UK

1‥Howlin@surrey.ac.uk Department of Chemistry, Faculty of Engineering and Physical Sciences, University of Surrey, Guildford, GU2 7XH, UK

${ }^{2}$ Long Term Development Laboratory, Becker Industrial Coatings Ltd, Goodlass Road, Speke, Liverpool L24 9HJ, UK

\section{Abstract}

Computational simulations can be used to save on both time and costs, complementing experimental work and providing further guidance. Immiscible polymer blends induce phase segregation, and in some cases can produce useful multicoat systems. This works uses a range of Molecular Dynamics Simulations methods, including an extended Flory Huggins Interaction Parameter $\chi$ to initially probe the interactions and miscibility between ester monomers commonly used in coil coatings. This work indicates that blends with similar backbone structures or "like with like" show increased miscibility and those with different structures lead to a large $\chi$ value and immiscibility. Further to this, polyester blends with different backbone structures have then been coarse grained with MARTINI beads and simulations of $10 \mu$ s have been run to identify the morphology of the blends at the mesoscopic level. Finally, the melamine crosslinker commonly used in polyester formulations has previously been shown to form agglomerates at higher melamine content, these agglomerates have been shown in atomistic simulations.

Keywords include: Coil Coatings, Molecular Dynamics, Flory Huggins, Coarse-grained

\section{Introduction}

Coil coatings are specially formulated coatings used to enhance the durability and protection of metal substrates used in a wide variety of areas including architecture, transport, household appliances, industry and packaging [1]. These coatings are complicated systems made up of various polymer resins as well as pigments, crosslinkers and other additives. Polyester resins are commonly used in coil coatings due to their excellent chemical and stain resistance as well as high strength and strain properties. These saturated polyesters are used as either the topcoat or primer and can be branched 
or linear depending upon the combination of dibasic acids and diols used. Recent work has looked to combine the traditional multicoat coil coating system into a one pot blend where the coating phase segregates out into required layers [2]. For phase segregation to occur, the polymer blend itself must be immiscible [3]. Research on immiscibility in polyester blends has included recycling PET blends used in thermoplastics [4], producing materials with varying functionality such as flame retardancy [5] and improved thermal properties [6]. Often polyesters blends are reactively blended to form new covalent bonds with other polymer functional groups to improve the immiscibility $[7,8]$. Blends of aromatic esters, Poly(ethylene terephthalate) (PET) with poly(trimethylene terephthalate) (PTT) and Poly(butylene terephthalate) (PBT) with (PTT) have shown to be miscible [6] using Differential Scanning Calorimetry. The immiscibility between linear and aromatic components has been shown in blends of high-density polyethylene (HDPE) and PET [9] where the blend possessed poor adhesion and mechanical properties due to the immiscibility.

Computational modelling can be used to save on both time and costs before sometimes difficult and speciality experimental work is carried out. Molecular dynamics treats atoms in a molecule as rubber balls of different sizes held together by springs of different lengths creating a dynamic system using Newton's equation. Varying force fields are used to explain the interactions $[10,11]$. This work looks at the miscibility of different polyester blends using the individual ester monomers with an extended Flory Huggins model (Equation 1). Here the interaction parameter $\chi$ between the esters is determined [12-14].

$$
\frac{\Delta G}{R T}=\frac{\phi A}{n_{A}} \ln \phi A+\frac{\phi B}{n_{B}} \ln \phi B+\chi \phi A \phi B
$$

Equation 1. Extended Flory Huggins Model, where $\Delta G$ = change in Gibbs Free Energy of Mixing, $R=$ Gas Constant,$T=$ Absolute Temperature, $\phi=$ Volume Fraction of Component $\mathrm{X}, \mathrm{n}_{\mathrm{A}}=$ Degree of Polymerisation of Component, $\mathrm{X}=$ Interaction Parameter.

The use of Molecular Dynamics to predict properties of polymer systems is common. Atomistic models have been used to determine the physical and mechanical properties of cyanurate polymer blends [15] as well as to understand a variety of polymer systems and blends, poly (2-6 dimethyl 1-4) phenylene ether [16] and poly (vinyl alcohol) with poly (methyl methacrylate) [17]. The interaction parameter $\chi$ determined from the extended Flory Huggins model (Equation 1) has been used to understand blends including poly (Lactide) with styrene co vinyl phenol copolymers where the number of vinyl phenol moieties needed for the blend to become miscible was determined [18].

Coarse-grained simulations group together atoms and allow for longer simulations with larger systems to be run in comparison to atomistic simulations [19]. Coarse-grained 
simulations often use the MARTINI forcefield [20] designed for biomolecules [21], where three to five heavy atoms are represented by a bead that is assigned a classification related to the chemistry of the atoms involved. Coarse-grained work has previously been carried out to determine the mechanical properties of a typical polyester resins used in coil coatings [22]. In this work coarse-grained polyesters have been produced to provide an insight into the phase separation of immiscible blends and the properties of the polyesters involved.

Hexa(methoxymethyl)melamine HMMM is used as a crosslinker in thermosetting coil coating formulations to provide a tough and durable coating with improved chemical, heat, wear and scratch resistance. Here, hydroxyl groups at the ends and branches of the polyesters react with via a trans-etherification mechanism with the methoxy groups in HMMM to form large covalent networks [23]. The HMMM crosslinker has shown to selfcondense [24] and form agglomerates of between 5-8 $\mu \mathrm{m}$ in polyester/HMMM formulations using Raman spectroscopy [25]. The formation of these agglomerates has been shown to be dependent upon the amount of HMMM in the formulation, via Dynamic Mechanical Analysis [26]. Much smaller HMMM agglomerates of around $5 \mathrm{~nm}$ have also been suggested using coarse grained simulations [22]. This work uses atomistic simulations to further identify the formation of these melamine agglomerates.

\section{Method}

\section{Molecular Dynamics}

Molecular dynamics allow for large complicated systems to be studied over set time periods. These simulations fall into two categories of either atomistic or coarse-grained methods, with the atomistic variety having a greater computational and time expense. Atomistic simulations provide information for specific interactions but are restricted to length scales of $1-100 \AA$ and time scales of $1 \mathrm{fs}-100 \mathrm{~ns}$ [27]. Coarse grained simulations allow for longer time lengths and larger polymer systems with improved computational efficiency. For this reason, atomistic simulations have been chosen to initially identify interactions between ester structures. It is expected that the phase segregation of polymer chains is time dependent, favouring coarse grained methods and simulations in the microsecond range [28-31]. Coarse grained methods have been used to ensure phase segregation can be seen at the mesoscale and atomistic simulations have been chosen for the somewhat simpler polyester melamine system.

Molecular dynamics simulations are carried out in a series of steps, including system preparation, minimisation/relaxation, equilibration and production [32]. Initially the system of interest is prepared and forcefields explaining the forces between atoms and 
molecules are specified. Following this the energy of the system is minimised used set algorithms that remove unwanted confirmations produced when building. To mimic experimental conditions, different thermodynamic ensembles are used included including NVT, keeping temperature constant, NPT keeping pressure constant and NVE, keeping energy constant.

\section{Interaction parameter $\chi$}

The Materials Studio Software [33] allows for the swift and straightforward determination of the Flory Huggins interaction parameter $\chi_{1}$, providing an understanding to the immiscibility of different polymer mixtures with the use of the inbuilt Blends Module. A combination of ester monomers where used in this part of the study, see Figure 1 . The ends of the molecules are determined as non-contact to produce polymer like interactions. The structure of the monomers has been initially optimized using the conjugate gradient algorithm to ensure the lowest energy confirmations using the Forcite Module. The DREIDING forcefield was used throughout the simulations [22].

(ethyl propyl decanedioate (ester c)




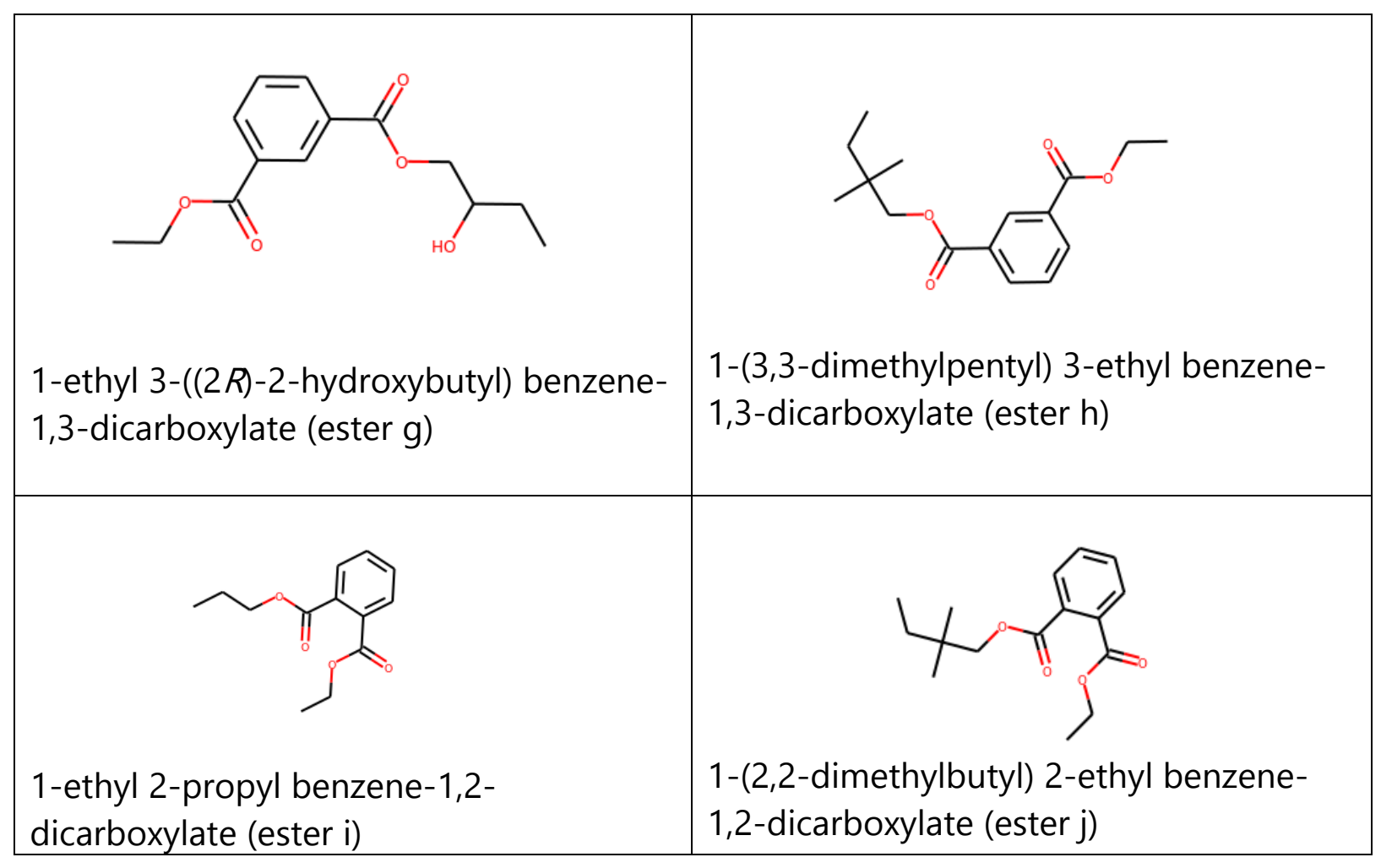

Figure 1. Ester monomers used in this study.

\section{Coarse-grained models}

Coarse grained simulations have been used due to the availability of increased time and length scales compared to atomistic simulations. Previous studies on polyester coil coating systems have applied the MARTINI coarse grained method to estimate the glass transition temperature of the polyester to within 30K of the experimental value [22]. GROMACS 2020 has been used to run the coarse-grained models, as these simulations are common practice with this software. In this work polyesters have been created with five repeating units of varying esters (Figure 2). Including the ester produced between meta-phtalic acid and neopentyl glycol (polyester 1). The ester produced between sebacic acid and ethylene glycol (polyester 2). The ester of para-phtalic acid and ethylene gylcol (polyester 3). The ester between adipic acid and neopentyl glycol (polyester 4). 


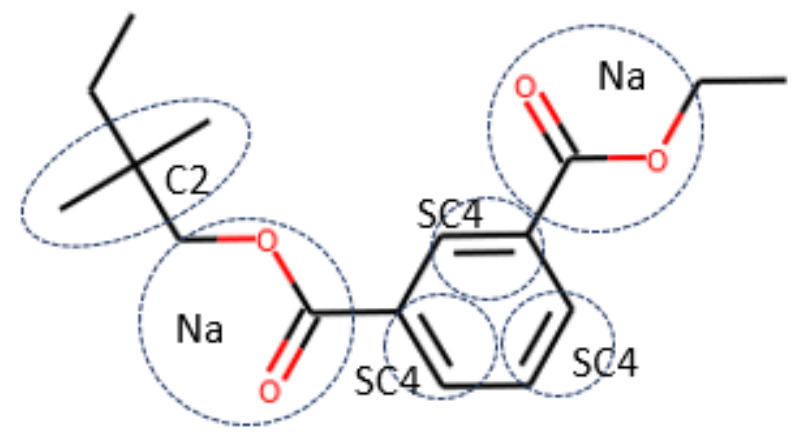

(polyester a) 1-(3-methoxy-2,2-dimethylpropyl) 3-methyl benzene-1,3-dicarboxylate

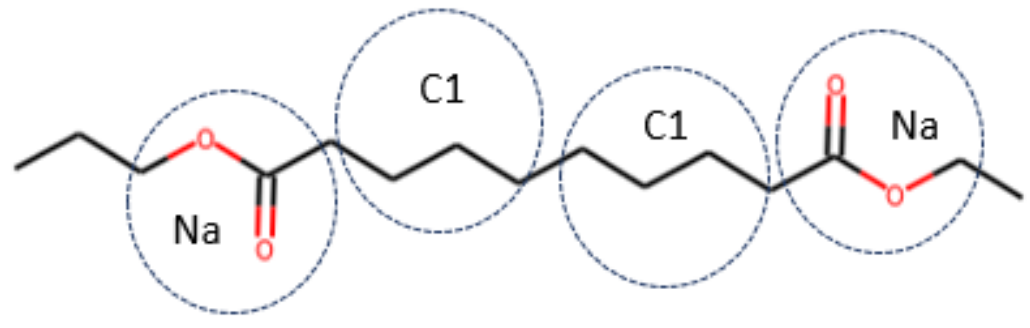

(polyester b) 1-O-methyl 10-O-propyl decanedioate

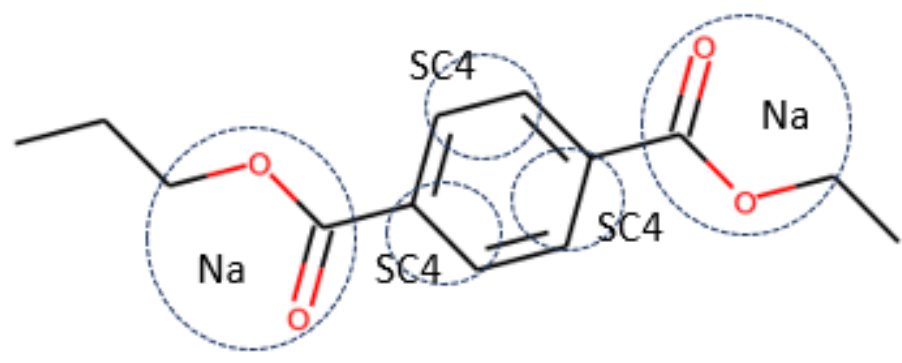

(polyester c) 1-O-methyl 4-O-propyl benzene-1,4-dicarboxylate

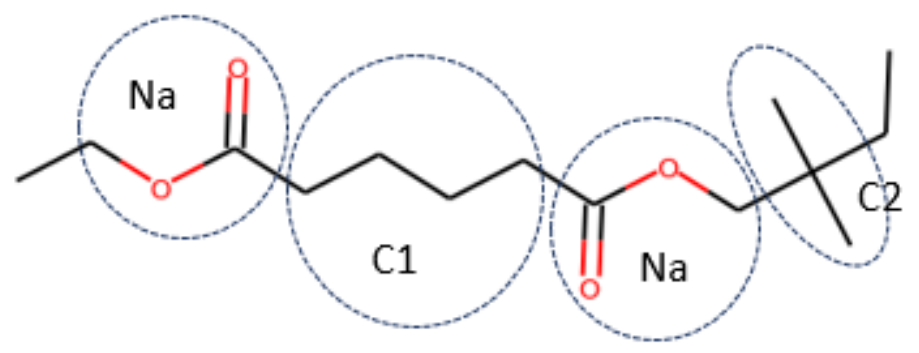

(polyester d) 2,2-dimethylbutyl methyl hexanedioate

Figure 2. MARTINI coarse grained beads used in this study 
Atomistic simulations of the polyesters have been initially carried out to determine the equilibrium values and force constants to be used for the coarse-grained models [11,34]. boxes of ten polyesters where created and simulations run using the Optimized Potentials for Liquid Simulations, all atom forcefield (OPLS-AA) [35]. The atomistic systems are optimised using the steepest descent algorithm removing unfavorable interactions produced when building the system. The NVT ensemble has been used to further relax the system keeping the number of particles, the volume of the system and the temperature constant [36]. The NPT ensemble [22], was used for 100 ns keeping the pressure and the temperature of the system constant

The MARTINI forcefield has been used for the coarse-grained simulations and the beads were mapped according to Figure 2 and are based upon previous work by Rossi et al breaking the polyester chain into chemical components determined by their polarity. The Python package PyCGTOOL developed at the University of Southampton has been used to map the atomistic simulations to coarse grained models [37]. Blends of the coarsegrained polyesters where produced with 10 chains of each polyester used to represent the blend and produce a system with a target density of $1 \mathrm{~g} \mathrm{~cm}^{-3}$. Initially these were optimised using the steepest descent algorithm to remove unfavourable interactions. These blends were then simulated for a total of $10 \mu \mathrm{s}$ using the NPT ensemble and the final trajectory was taken [22]. A generalized scheme of preparing the coarse-grained models is shown in Figure 3. 
a. Generation of input files, structure and topology

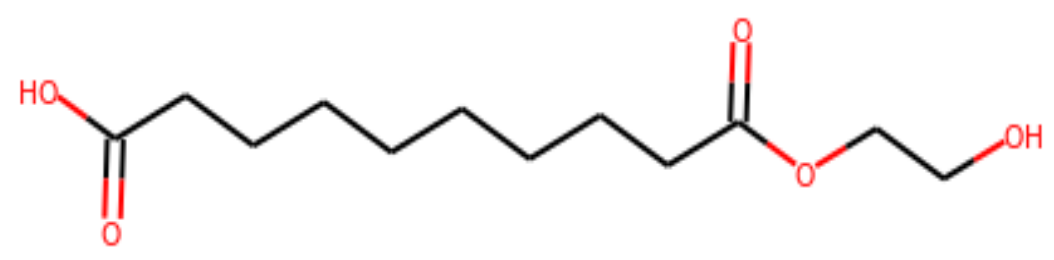

b. Atomistic simulations to generate the parameters for coarse-grained simulations.

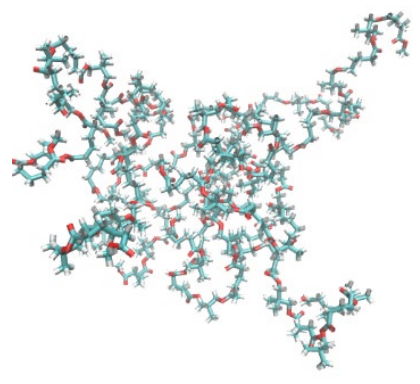

c. Determine MARTINI coarse grain beads, dependent upon chemistry

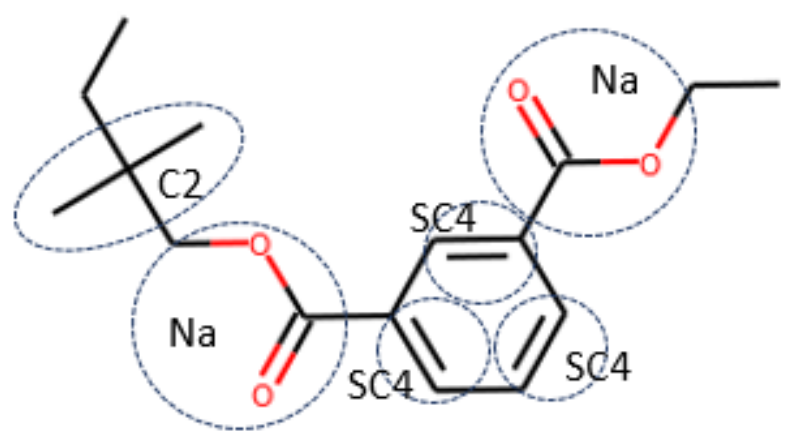

d. Simulations of the coarse grain beads

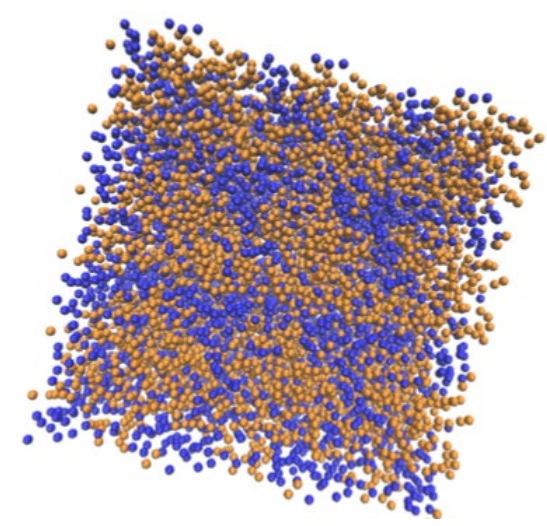

Figure 3. Method for producing coarse-grained polymer blends 


\section{Melamine clusters}

To investigate the formation of melamine agglomerates with increasing melamine content, atomistic simulations have been used. Materials Studio allows for the straightforward development of various polymer systems with a graphical user interface. The Amorphous Cell Module was used to build the polyester and melamine system at different concentrations. The Forcite Module has been used to optimise and run the simulations. The UNIVERSAL forcefield was used throughout the simulations [38]. A polyester made up of neopentyl glycol, ortho-phthalic acid, adipic acid and a triol was built along with the HMMM molecule. The structures of the molecules were initially optimised using the Smart algorithm.

Agglomerates of HMMM crosslinker have been shown to form with increasing melamine content [26], and for this reason unit cells of the polyester with increasing HMMM content where produced, in this case 5, 10, 15, 20, 25, 30\% HMMM. A nominal density of $1 \mathrm{~g} \mathrm{~cm}$ ${ }^{3}$ was used for each and the unit cells where initially minimised with the Smart algorithm. The NVT ensemble was used to further optimise the cell for $10 \mathrm{ps,} \mathrm{minimizing} \mathrm{the} \mathrm{energy}$ of the system. Before continuing to use the NPT ensemble with the Andersen Thermostat and Beresden Barostat for 10ps with a time step of $2 \mathrm{fs}$. Finally, the NVE ensemble was used for 500ps to give the final system trajectory. These simulation times were chosen as a compromise of both simulation length providing accuracy, and experimental constraints. 


\section{Results}

\section{Interaction parameter $\chi$}

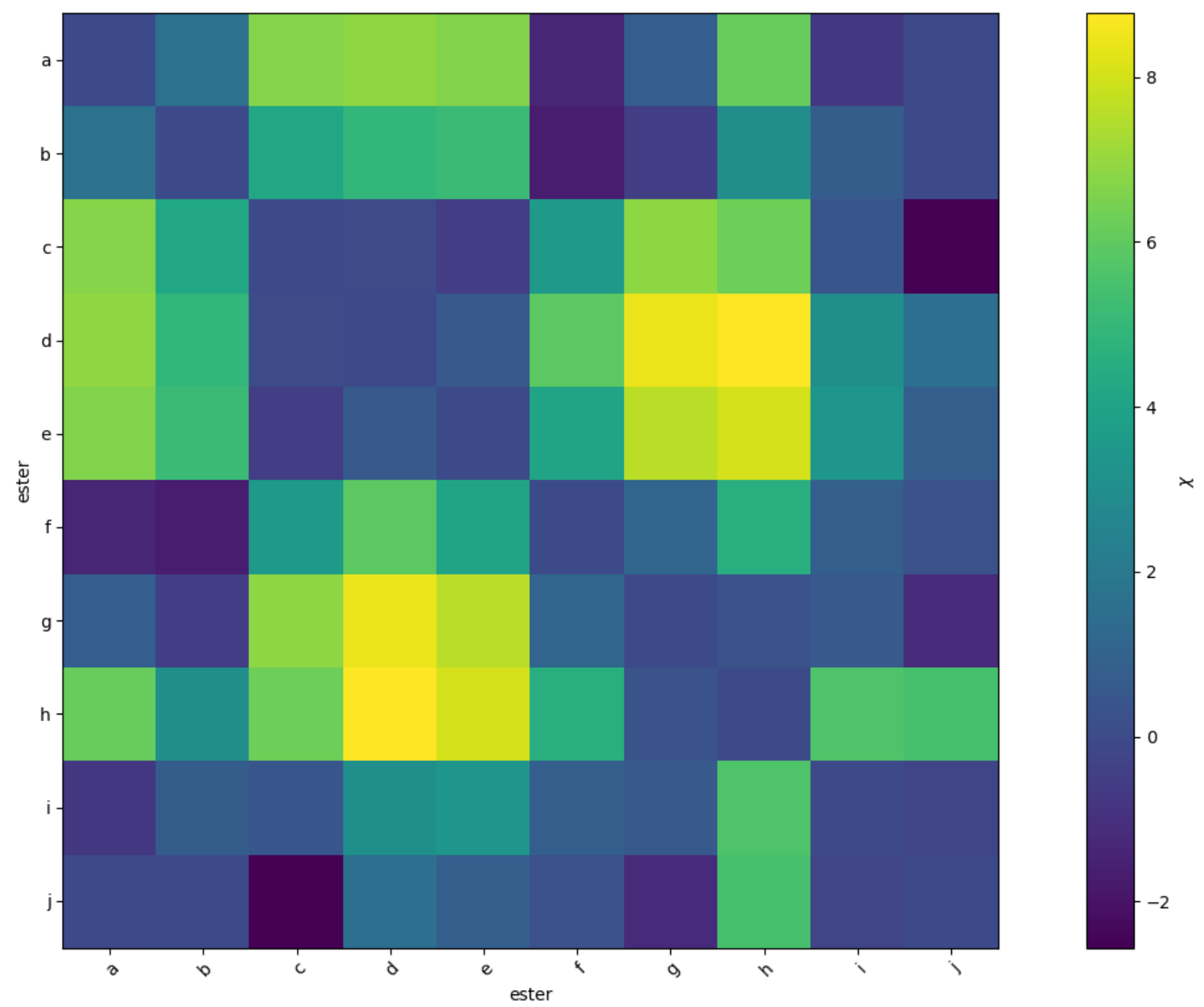

Figure 4. $\chi$ interaction parameter for ester-ester interactions

The interaction parameter $\chi$ for different ester-ester combinations is shown in Figure 4. Blends that have a large $\chi$ value are generally immiscible and those with a small or negative $\chi$ value are miscible [2]. Pairs of dimers that are made up of a similar backbone tend to be miscible with each other and show small or negative $\chi$ values. The aromatic dimers commonly make miscible blends with other aromatic dimers and linear structures with other linear chains.

Adipic acid based esters (ester $\mathrm{d}$ and e, see Figure 4) in combination with aromatic based esters (ester $a, b, g$ and $h$ ) have shown to produce the most immiscible blends. There are several ester combinations that do not follow the aromatic-aliphatic immiscibility. The 
aromatic polyester ortho phtalic acid with neopentyl glycol (ester j) forms blends with a small to negative $\chi$ value for most esters, this is the same for the ester of ortho phtalic acid with ethylene glycol (ester i). Esters made up of the same carboxylic acid, as with para phtalic acid with neopentyl glycol (ester b) and para phtalic acid with ethylene glycol (ester a) show similar results indicating that the choice of carboxylic acid determines the immiscibility.

The prediction of the $\chi$ parameter is difficult [39], unfavoured interactions along a chain can together produce a entropically more favoured system. With chemically similar polymers, as with this study. The entropic component of $\chi$, due to unfavourable packing is significant. This can be understood in this work as to the comparison of the aliphatic and aromatic constituents.

\section{Coarse-grained simulations}

From the earlier section looking at the interaction parameter it was indicated the structure of the backbone determined the miscibility of the blend. The blends of polyester $d$ and polyester $\mathrm{a}$, Figure 5 and polyester $\mathrm{d}$ with polyester e, Figure 6 are made up of dissimilar backbone structures and are expected to be immiscible. These coarse-grained models show regions of agglomerates of each of the polyesters.

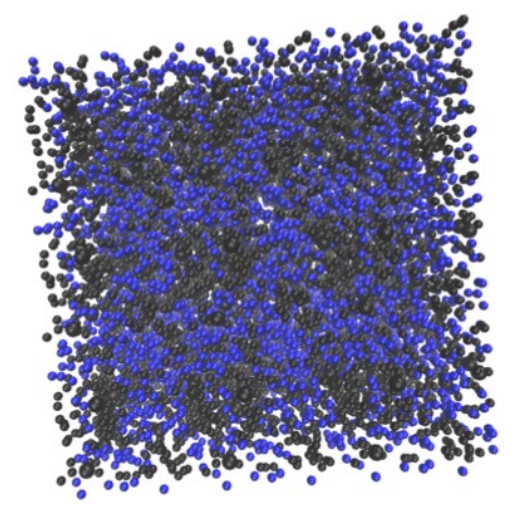

Figure 5. polyester $d=$ blue, polyester $a=$ black

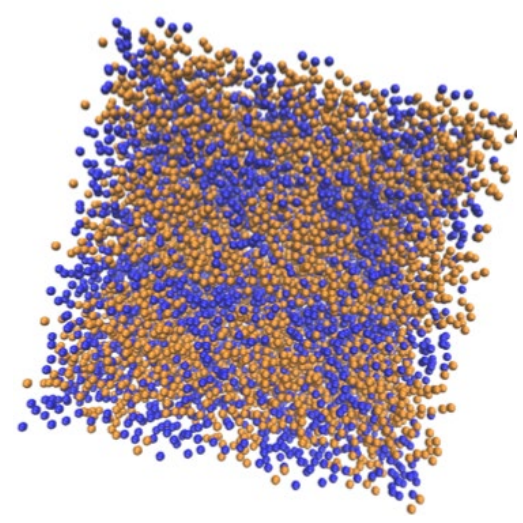

Figure 6 . polyester $d=$ blue, polyester $c=$ orange 
The polyester blend shown in Figure 7 is made up of a similar aliphatic backbone structure consisting of polyester adipic acid and neopentyl glycol with sebacic acid and ethylene glycol. In comparison with the previous coarse-grained structures this produces a layered structure.

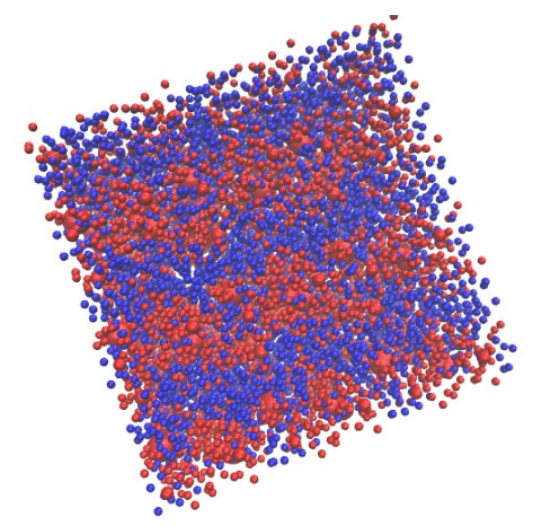

Figure 7. polyester $d=$ blue, polyester $b=$ red

The radius of gyration $\left(R_{g}\right)$, seen in Equation 2. Explains the compactness of a polymer, see Figure 8 . This is often used to understand the favourability of inter and intra molecular interactions of a polymer [40]. The $R_{g}$ of the different polyesters remain constant throughout each of the simulations. However, there is a difference in $R_{g}$ between the polyester blends of similar backbone and those of not, see Figure 9 .

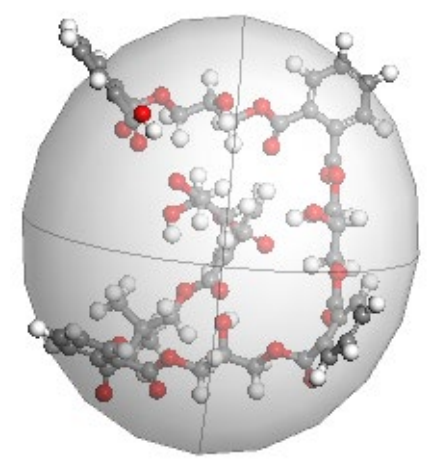

Figure 8. Radius of gyration

$$
\mathrm{R}_{\mathrm{g}}=\left(\frac{\sum_{\mathrm{i}}\left|\mathrm{r}_{\mathrm{i}}\right|^{2} \mathrm{~m}_{\mathrm{i}}}{\sum_{\mathrm{i}} \mathrm{m}_{\mathrm{i}}}\right)^{\frac{1}{2}}
$$

Equation 2. Radius of gyration, here $m_{i}$ is the mass of atom $i$ and $\mathbf{r}_{i}$ the position of atom $i$ with respect to the center of mass of the molecule 


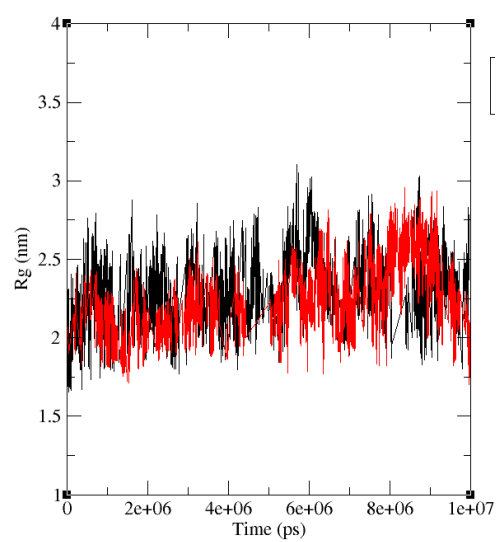

(A)

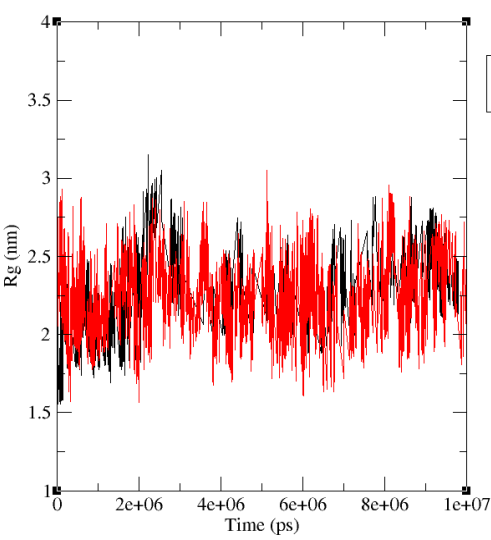

(B)

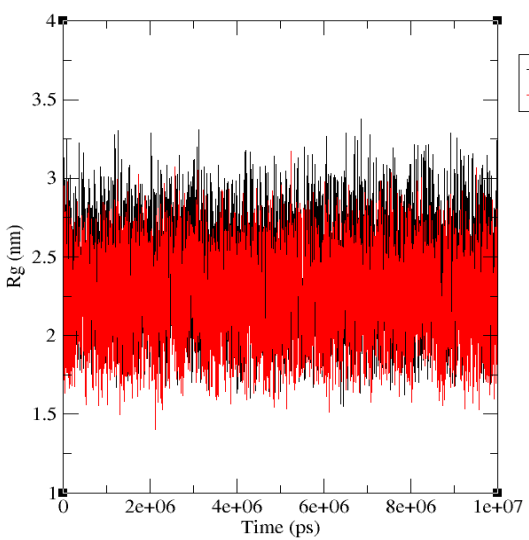

(C)

Figure 9. Radius of gyration, (A) polyester $d$ with $a_{1}(B)$ polyester $d$ with $c_{1}(C)$ polyester $d$ with $b$, polyester $d$ is shown in red.

\section{Crosslinker agglomerates}

The final trajectory file has been taken from the simulations and visualized using the Atomic Simulation Environment [41]. Grey, red and white atoms make up the polyester, the blue atoms show the ring of the melamine.

At crosslinker concentrations of 5 and $10 \%$, see Figures 10.1,10.2. The HMMM molecules can be seen to be distant from each other and possible interactions are diminished. At 15, $20 \%$ crosslinker the HMMM molecules have moved closer in contact. For increased melamine content at 25 and $30 \%$, the HMMM molecules have shown to produce clusters apart from the polyester chain. Upon inspection of Figure 10.4 with $20 \%$ of HMMM, the molecular interactions are between the ether groups in the crosslinkers rather than between the triazine rings. This attraction provides the potential for self-condensation to occur between the molecules and for the growth of HMMM agglomerates. 


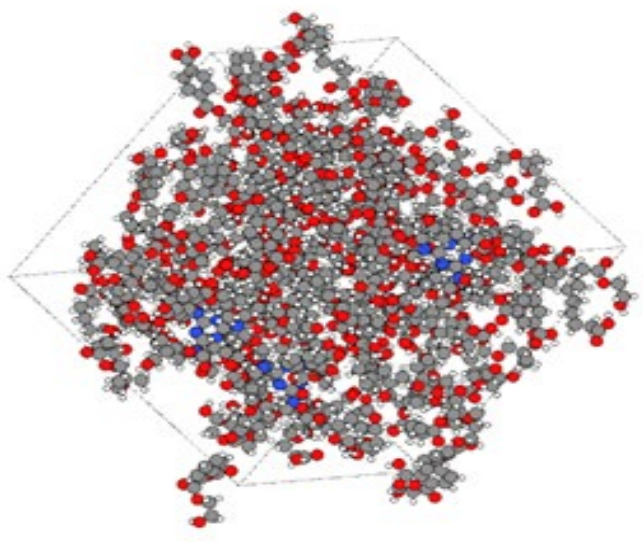

Figure 10.1 Unit cell of 5\% HMMM

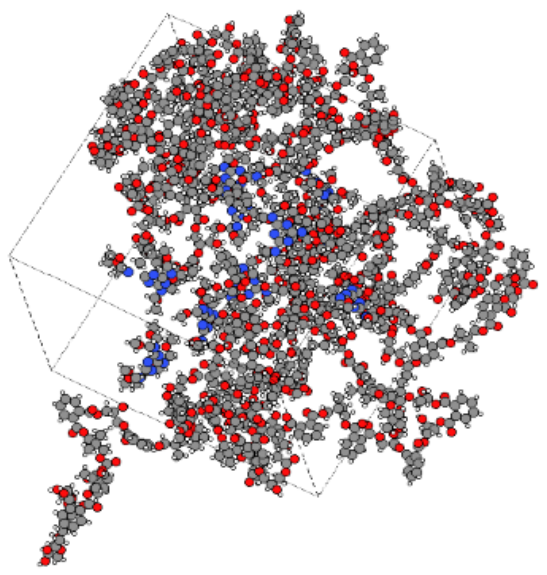

Figure 10.3 Unit cell of 15\% HMMM

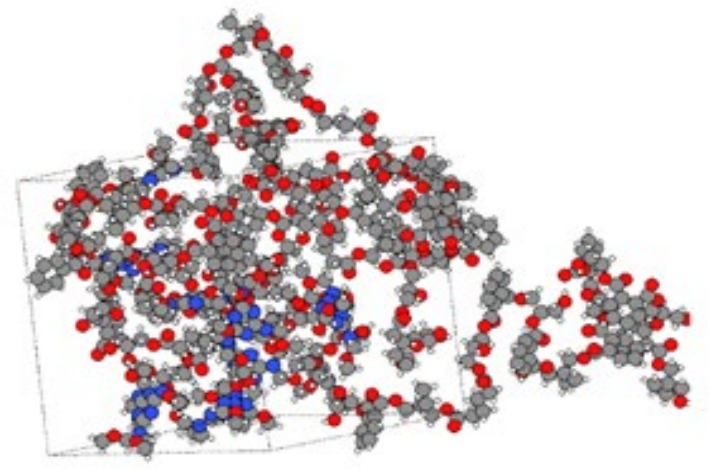

Figure 10.5 Unit cell of $25 \%$ HMMM

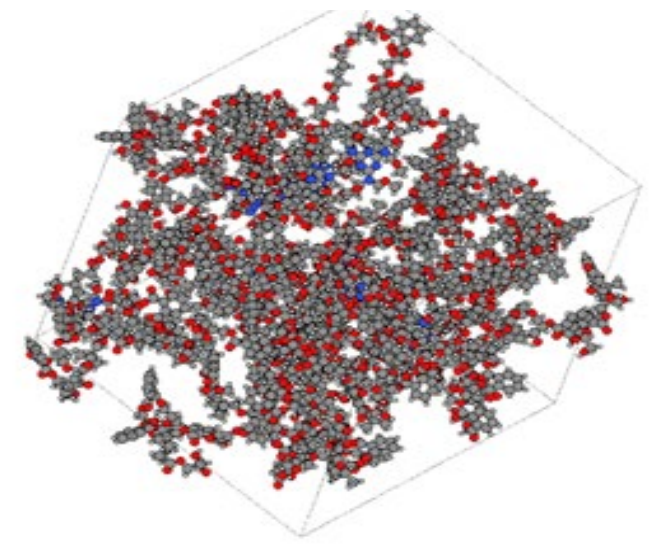

Figure 10.2 10\% Unit cell of HMMM

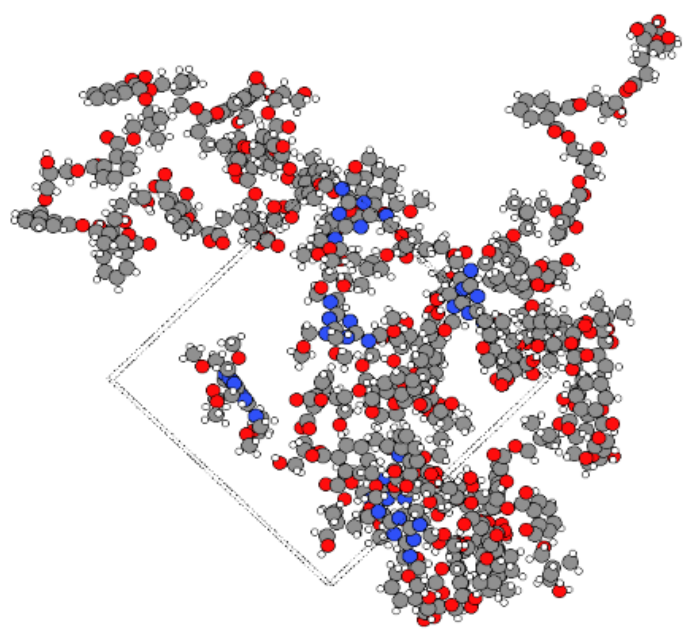

Figure 10.4 20\% Unit cell of HMMM

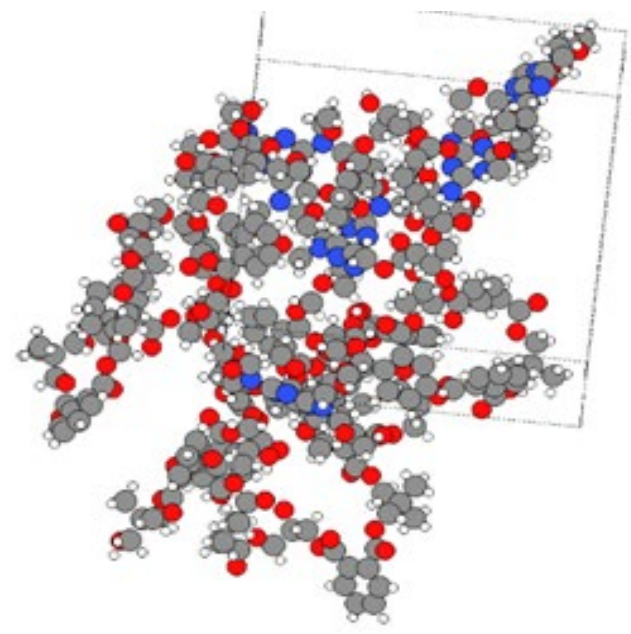

Figure 10.6 Unit cell of $30 \%$ HMMM 


\section{Discussion}

\section{Interaction parameter $\chi$}

The results shown in Figure 4 follow the general idea that 'like dissolves like,' where esters with similar chemical characteristics are generally miscible with each other due to increased favourable intermolecular interactions. However, several exclusions from this rule are seen including the linear ester $j$, producing miscible blends with almost all other esters. The linearity of this ester allows more positions of favourable interactions to be produced along the ester than in some of the other aromatic esters with different structures. Ester h produces the most immiscible ester blends and is made up of aromatic carboxylic acid with the carbonyl groups at the meta position, indicating that the position of the carbonyl group affects the ester interactions and ultimately the miscibility of the blends. Ester $\mathrm{g}$ has the additional functionality of the electron donating $-\mathrm{OH}$ bond, this has not improved the bonding and compatibility towards aliphatic components. Changing the backbone structure has shown to produce changes in both the $\mathrm{Tg}$ and mechanical properties of the resin[42].

The miscibility of polymers with similar structures has been seen before, aliphatic polymer blends made up of polyvinylidene difluoride (PVDF) and poly(methyl methacrylate) (PMMA) show a negative Flory Huggins interaction parameter indicating miscibility $[43,44]$. However, examples of immiscibility created in polymers of the same structure do exist, including blends of poly(ethylene) (PE) and poly(propylene) (PP) that have shown to be thermodynamically immiscible [36]. The immiscibility of these blends is attributed to the lack of specific interactions and polarity between the blends.

Noncovalent interactions are commonly recognised in biological and pharmocological systems $[45,46]$. Ester-ester interactions include dispersion forces with and dipole-dipole interactions between carbonyl groups [47-49], additionally the aromatic component included in some of the monomers provides the functionality for bonding between aromatic rings, termed pi-stacking [50]. Research compared aliphatic and aromatic molecules and showed that for molecules larger than napthalene, possible pi-stacking interactions exist in aromatic dimers [51]. These interactions have shown to be either a sandwich structure seen in benzene or parallel displaced as seen in aromatics with substituents [52], Figure 11 shows the lowest energy confirmation of the dimer between ester $b$ with ester $f$ indicating this parallel displaced structure between the two aromatic rings. 


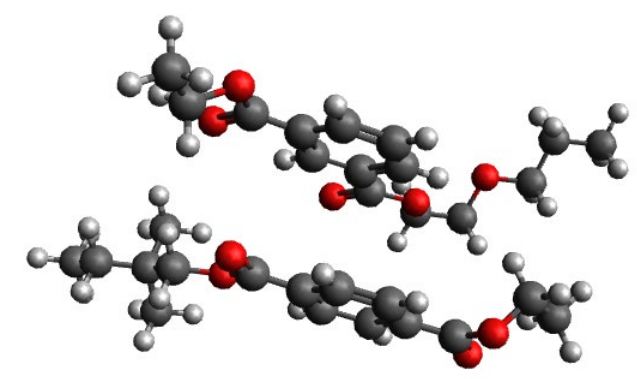

Figure 11. Pi-stacking shown with ester $b$ with ester $f$

\section{Coarse-grained simulations}

The use of coarse-grained models has provided an insight into the structure of the polyester blends at the mesoscopic scale. It has allowed the original properties of the atomistic simulations to be mapped onto coarse grained models providing a less intensive computation. The blends made up with varying polyester structures have produced agglomerates, in comparison with those of the same structure that has produced a layered structure, see Figure 9. For the aim of this work to look at polyester blends that produce phase segregated structures this combination of aliphatic-aliphatic ester blend is of note. This blend gave an interaction parameter value of 0.6 , indicating that the level of immiscibility determines the structure produced.

The radius of gyration $\left(R_{g}\right)$ has often been used to show changes in polymer structures and biological systems throughout a simulation $[53,54]$ and is a property that may be experimentally derived. The radius of gyration shown in Figure 9, for each of the polyesters compares with previous values determined for similar polyesters [22]. Further work should look to determine experimental values such as the glass transition temperature and tensile tests to validate simulations $[15,22]$

\section{Crosslinker agglomerates}

The formation of HMMM crosslink agglomerates has been shown experimentally using Dynamic Mechanical Analysis (DMA) [55]. Here an oscillating force is applied, and the strain response of the material is measured over a range of temperatures. Due to the viscoelastic nature of polymers, a lag in the strain response is created and from that the storage modulus ( $\left.E^{\prime}\right)$ and the loss modulus ( $\left.E^{\prime \prime}\right)$ is determined [56]. The storage modulus shows the stored elastic energy, and the loss modulus represents the energy which is dissipated as heat [24]. The ratio of the two is taken, shown in Equation 3 and the magnitude of the tangent represents the damping of the material. Figure 12, shows 
symmetric peaks for tan $(\delta)$ increasing related to the melamine content, but at $30 \%$ melamine content a shoulder is formed on the left of the original peaks and is proposed as the formation of a second network of self-condensed melamine [26].

$$
\tan (\delta)=E^{\prime \prime} / E^{\prime}
$$

Equation 3. $\tan (\delta)$, the damping of the material is represented as the ratio of the storage modulus $\mathrm{E}^{\prime}$ and the loss modulus $\mathrm{E}^{\prime \prime}$.

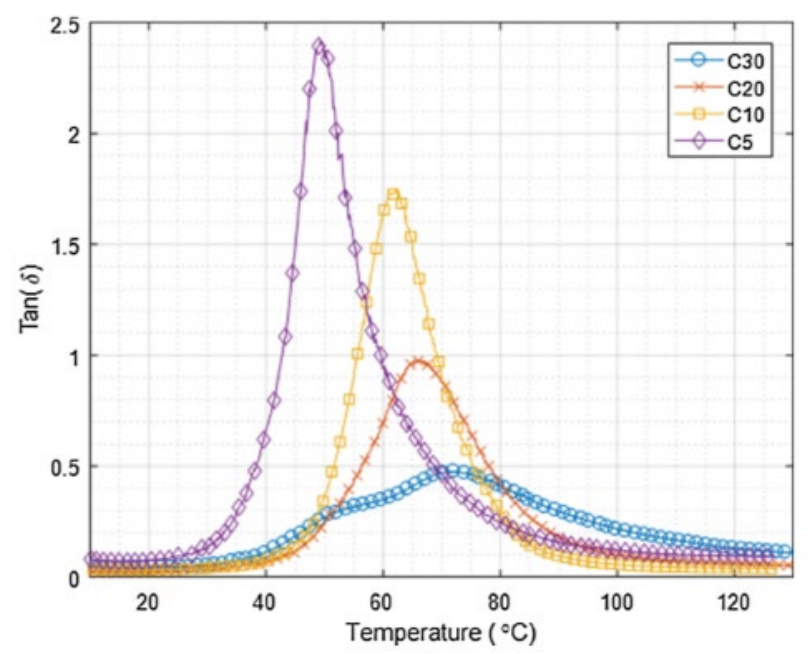

Figure 12. Dynamic mechanical analysis showing effect of crosslinker content on tan $(\delta)$ versus temperature [26]

\section{Conclusion}

Molecular Dynamics has shown to be a powerful tool to determine the interaction parameter $\chi$ of numerous ester blends, giving an insight into the miscibility of combinations of esters with different backbone structures, providing means for further experimental work and formulation advice. Work using an extended Flory Huggins method has shown that blends of linear and aromatic esters create immiscibility in a blend. Coarse grained simulations have been run for $10 \mu$ s showing variations in morphology due to changes in polyester backbone structure. For future work on coarse grained simulations, the inclusion of experimental observables such as glass transition temperature should be used to validate simulations. Atomistic simulations have however complimented previous experimental work showing the formation melamine agglomerates at higher concentrations and provided and understanding of the interactions between them. 
[1] J. Sander, Coil Coating, Vincentz Network, Hanover 2014, Vincentz Network, 2014.

[2] A. Beaugendre, S. Degoutin, S. Bellayer, C. Pierlot, S. Duquesne, M. Casetta, M. Jimenez, Self-stratifying coatings: A review, Progress in Organic Coatings. 110 (2017) 210-241. https://doi.org/10.1016/j.porgcoat.2017.03.011.

[3] Vladimir V. Verkholantsev, Exploiting self-stratification, European Coatings Journal,Vincentz Network +++ Schiffgraben 43, D-30175 Hannover. (2005) 20-20.

[4] D. Pospiech, L. Häußler, A. Korwitz, O. Fischer, S. Starke, D. Jehnichen, T. Köppl, V. Altstädt, The miscibility of poly(butylene terephthalate) (PBT) with phosphorus polyester flame retardants, High Performance Polymers. 24 (2012). https://doi.org/10.1177/0954008311431116.

[5] S.-S. Lee, H.M. Jeong, J.Y. Jho, T.O. Ahn, Miscibility of poly(ethylene terephthalate)/poly(estercarbonate) blend, Polymer 41. (2000) 1773-1782.

[6] N. Torres, J.J. Robin, B. Boutevin, Study of compatibilization of HDPE-PET blends by adding grafted or statistical copolymers, Journal of Applied Polymer Science. 81 (2001). https://doi.org/10.1002/app.1678.

[7] G. Aravinthan, D.D. Kale, Blends of poly(ethylene terephthalate) and poly(butylene terephthalate), Journal of Applied Polymer Science. 98 (2005). https://doi.org/10.1002/app.22017.

[8] Y.-H. Kuo, E.M. Woo, Miscibility in Two Blend Systems of Homologous Semicrystalline Aryl Polyesters Involving Poly(trimethylene terephthalate), 2003.

[9] S. Aid, A. Eddhahak, S. Khelladi, Z. Ortega, S. Chaabani, A. Tcharkhtchi, On the miscibility of PVDF/PMMA polymer blends: Thermodynamics, experimental and numerical investigations, Polymer Testing. 73 (2019).

https://doi.org/10.1016/j.polymertesting.2018.11.036.

[10] A.K. Rappe, C.J. Casewit, K.S. Colwell, W.A. Goddard, W.M. Skiff, UFF, a full periodic table force field for molecular mechanics and molecular dynamics simulations, Journal of the American Chemical Society. 114 (1992). https://doi.org/10.1021/ja00051a040.

[11] W.L. Jorgensen, D.S. Maxwell, J. Tirado-Rives, Development and Testing of the OPLS All-Atom Force Field on Conformational Energetics and Properties of Organic Liquids, Journal of the American Chemical Society. 118 (1996). https://doi.org/10.1021/ja9621760. 
[12] C.F. Fan, B.D. Olafson, M. Blanco, S.L. Hsu, Application of molecular simulation to derive phase diagrams of binary mixtures, Macromolecules. 25 (1992) 3667-3676. https://doi.org/10.1021/ma00040a010.

[13] Paul J. Flory, Principles of Polymer Chemistry, Ithaca Cornell University Press, 1953.

[14] J.S. Higgins, J.E.G. Lipson, R.P. White, A simple approach to polymer mixture miscibility, Philosophical Transactions of the Royal Society A: Mathematical, Physical and Engineering Sciences. 368 (2010). https://doi.org/10.1098/rsta.2009.0215.

[15] A. Crawford, I. Hamerton, G. Cavalli, B. Howlin, Quantifying the Effect of Polymer Blending through Molecular Modelling of Cyanurate Polymers, PloS One. 7 (2012) e44487. https://doi.org/10.1371/journal.pone.0044487.

[16] H. Wang, B. Shentu, R. Faller, Refinement of a coarse-grained model of poly(2,6dimethyl-1,4-phenylene ether) and its application to blends of PPE and PS, Molecular Simulation. 42 (2016) 312-320. https://doi.org/10.1080/08927022.2015.1047368.

[17] S.S. Jawalkar, S.G. Adoor, M. Sairam, M.N. Nadagouda, T.M. Aminabhavi, Molecular Modeling on the Binary Blend Compatibility of Poly(vinyl alcohol) and Poly(methyl methacrylate): An Atomistic Simulation and Thermodynamic Approach, The Journal of Physical Chemistry B. 109 (2005) 15611-15620. https://doi.org/10.1021/jp051206v.

[18] I.M. de Arenaza, E. Meaurio, J.-R. Sarasu, Analysis of the Miscibility of Polymer Blends Through Molecular Dynamics Simulations, Polymerization. (2012). https://doi.org/10.5772/51327.

[19] T.E. Gartner, A. Jayaraman, Modeling and Simulations of Polymers: A Roadmap, Macromolecules. 52 (2019). https://doi.org/10.1021/acs.macromol.8b01836.

[20] S.J. Marrink, H.J. Risselada, S. Yefimov, D.P. Tieleman, A.H. de Vries, The MARTINI Force Field: Coarse Grained Model for Biomolecular Simulations, The Journal of Physical Chemistry B. 111 (2007) 7812-7824. https://doi.org/10.1021/jp071097f.

[21] H. Yagyu, J.-Y. Lee, D.-N. Kim, O. Tabata, Coarse-Grained Molecular Dynamics Model of Double-Stranded DNA for DNA Nanostructure Design, The Journal of Physical Chemistry B. 121 (2017). https://doi.org/10.1021/acs.jpcb.7b03931. 
[22] G. Rossi, I. Giannakopoulos, L. Monticelli, N. Rostedt, S. Puisto, C. Lowe, A. Taylor, I. Vattulainen, T. Ala-Nissila, A MARTINI coarse-grained model of a thermoset polyester coating, Macromolecules. 44 (2011) 6198-6208. https://doi.org/10.1021/ma200788a.

[23] T. Greunz, C. Lowe, E. Bradt, S. Hild, B. Strauß, D. Stifter, A study on the depth distribution of melamine in polyester-melamine clear coats, Progress in Organic Coatings. 115 (2018) 130-137. https://doi.org/10.1016/j.porgcoat.2017.11.014.

[24] N.J.W. Gamage, D.J.T. Hill, C.A. Lukey, P.J. Pomery, Thermal characterization of polyester-melamine coating matrices prepared under nonisothermal conditions, Journal of Polymer Science Part A: Polymer Chemistry. 41 (2003). https://doi.org/10.1002/pola.10698.

[25] W. Zhang, R. Smith, C. Lowe, Confocal Raman microscopy study of the melamine distribution in polyester-melamine coil coating, Journal of Coatings Technology and Research. 6 (2009) 315-328. https://doi.org/10.1007/s11998-0089136-2.

[26] F.S. Sorce, S. Ngo, C. Lowe, A.C. Taylor, The effect of HMMM crosslinker content on the thermal-mechanical properties of polyester coil coatings, Progress in Organic Coatings. 137 (2019). https://doi.org/10.1016/j.porgcoat.2019.105338.

[27] T.E. Gartner, A. Jayaraman, Modeling and Simulations of Polymers: A Roadmap, Macromolecules. 52 (2019) 755-786.

https://doi.org/10.1021/acs.macromol.8b01836.

[28] U. Kapoor, A. Kulshreshtha, A. Jayaraman, Development of Coarse-Grained Models for Poly(4-vinylphenol) and Poly(2-vinylpyridine): Polymer Chemistries with Hydrogen Bonding, Polymers. 12 (2020). https://doi.org/10.3390/polym12112764.

[29] T. Ohkuma, K. Kremer, A composition transferable and time-scale consistent coarse-grained model for cis-polyisoprene and vinyl-polybutadiene oligomeric blends, Journal of Physics: Materials. 3 (2020). https://doi.org/10.1088/25157639/ab906b.

[30] Q. Sun, R. Faller, Systematic Coarse-Graining of a Polymer Blend: Polyisoprene and Polystyrene, Journal of Chemical Theory and Computation. 2 (2006). https://doi.org/10.1021/ct600065v. 
[31] B. Mantisi, A. Tanguy, G. Kermouche, E. Barthel, Atomistic response of a model silica glass under shear and pressure, The European Physical Journal B. 85 (2012). https://doi.org/10.1140/epjb/e2012-30317-6.

[32] BIOVIA, Materials Studio, Accelrys.com. Available: http://accelrys.com/products/ materials-studio/. Accessed 14, March 2012, BIO. (n.d.).

[33] S.L. Mayo, B.D. Olafson, W.A. Goddard, DREIDING: a generic force field for molecular simulations, The Journal of Physical Chemistry. 94 (1990) 8897-8909. https://doi.org/10.1021/j100389a010.

[34] M. YABE, K. MORI, K. UEDA, M. TAKEDA, Development of PolyParGen Software to Facilitate the Determination of Molecular Dynamics Simulation Parameters for Polymers, Journal of Computer Chemistry, Japan -International Edition. 5 (2019). https://doi.org/10.2477/jccjie.2018-0034.

[35] E. Braun, J. Gilmer, H.B. Mayes, D.L. Mobley, J.I. Monroe, S. Prasad, D.M. Zuckerman, Best Practices for Foundations in Molecular Simulations [Article v1.0], Living Journal of Computational Molecular Science. 1 (2019). https://doi.org/10.33011/livecoms.1.1.5957.

[36] H.J.C. Berendsen, J.P.M. Postma, W.F. van Gunsteren, A. DiNola, J.R. Haak, Molecular dynamics with coupling to an external bath, The Journal of Chemical Physics. 81 (1984). https://doi.org/10.1063/1.448118.

[37] W. Humphrey, A. Dalke, K. Schulten, VMD: Visual molecular dynamics, Journal of Molecular Graphics. 14 (1996). https://doi.org/10.1016/0263-7855(96)00018-5.

[38] J.S. Higgins, J.E.G. Lipson, R.P. White, A simple approach to polymer mixture miscibility, Philosophical Transactions of the Royal Society A: Mathematical, Physical and Engineering Sciences. 368 (2010). https://doi.org/10.1098/rsta.2009.0215.

[39] D. Kozuch, W. Zhang, S. Milner, Predicting the Flory-Huggins $\chi$ Parameter for Polymers with Stiffness Mismatch from Molecular Dynamics Simulations, Polymers. 8 (2016). https://doi.org/10.3390/polym8060241.

[40] A.H. Larsen, J.J. Mortensen, J. Blomqvist, I.E. Castelli, R. Christensen, Marcin Dułak, J. Friis, M.N. Groves, B. Hammer, C. Hargus, E.D. Hermes, P.C. Jennings, P.B. Jensen, J. Kermode, J.R. Kitchin, E.L. Kolsbjerg, J. Kubal, Kristen Kaasbjerg, S. Lysgaard, J.B. Maronsson, T. Maxson, T. Olsen, L. Pastewka, Andrew Peterson, C. Rostgaard, J. Schiøtz, O. Schütt, M. Strange, K.S. Thygesen, Tejs Vegge, L. 
Vilhelmsen, M. Walter, Z. Zeng, K.W. Jacobsen, The atomic simulation environment-a Python library for working with atoms, Journal of Physics: Condensed Matter. 29 (2017) 273002. http://stacks.iop.org/0953$8984 / 29 / \mathrm{i}=27 / \mathrm{a}=273002$.

[41] F. Fraïsse, V. Verney, S. Commereuc, M. Obadal, Recycling of poly(ethylene terephthalate)/polycarbonate blends, Polymer Degradation and Stability. 90 (2005). https://doi.org/10.1016/j.polymdegradstab.2005.02.019.

[42] I. Giannakopoulos, The mechanical properties of polyester-based coil coatings. Correlations with chemical structure, 2012.

[43] D. Leckband, J. Israelachvili, Intermolecular forces in biology, Quarterly Reviews of Biophysics. 34 (2001). https://doi.org/10.1017/S0033583501003687.

[44] M. Maniruzzaman, D.J. Morgan, A.P. Mendham, J. Pang, M.J. Snowden, D. Douroumis, Drug-polymer intermolecular interactions in hot-melt extruded solid dispersions, International Journal of Pharmaceutics. 443 (2013). https://doi.org/10.1016/j.ijpharm.2012.11.048.

[45] E. Saiz, J.P. Hummel, P.J. Flory, M. Plavsic, Direction of the dipole moment in the ester group, The Journal of Physical Chemistry. 85 (1981). https://doi.org/10.1021/j150622a002.

[46] E. Galbiati, M. del Zoppo, G. Tieghi, G. Zerbi, Dipole-dipole interactions in simple esters and in liquid-crystal polyesters, Polymer. 34 (1993) 1807-1810.

[47] T. Nakano, Synthesis, structure and function of $\pi$-stacked polymers, Polymer Journal. 42 (2010) 103-123. https://doi.org/10.1038/pj.2009.332.

[48] H. KAJI, N. INUI, F. HORII, Analysis of \&pi;-Stacking Structure in Amorphous PET by Two-Dimensional Double-Quantum Solid-State NMR Spectroscopy, Polymer Preprints, Japan. 52 (2003) 682.

[49] W. Chen, K. Wu, Z. Qu, M. Lu, Intrinsic high thermal conductive co-polyester based on offset $\pi-\pi$ stacking, European Polymer Journal. 121 (2019). https://doi.org/10.1016/j.eurpolymj.2019.109275.

[50] S. Grimme, Do Special Noncovalent $\pi-\pi$ Stacking Interactions Really Exist?, Angewandte Chemie International Edition. 47 (2008).

https://doi.org/10.1002/anie.200705157. 
[51] S.E. Wheeler, Understanding substituent effects in noncovalent interactions involving aromatic rings, Accounts of Chemical Research. 46 (2013) 1029-1038. https://doi.org/10.1021/ar300109n.

[52] G. Yao, J. Zhao, S.B. Ramisetti, D. Wen, Atomistic Molecular Dynamic Simulation of Dilute Poly(acrylic acid) Solution: Effects of Simulation Size Sensitivity and Ionic Strength, Industrial and Engineering Chemistry Research. 57 (2018) 17129-17141. https://doi.org/10.1021/acs.iecr.8b03549.

[53] H. Hashemzadeh, H. Javadi, M.H. Darvishi, Study of Structural stability and formation mechanisms in DSPC and DPSM liposomes: A coarse-grained molecular dynamics simulation, Scientific Reports. 10 (2020). https://doi.org/10.1038/s41598020-58730-z.

[54] D.G. Mintis, V.G. Mavrantzas, Effect of $\mathrm{pH}$ and Molecular Length on the Structure and Dynamics of Short Poly(acrylic acid) in Dilute Solution: Detailed Molecular Dynamics Study, The Journal of Physical Chemistry B. 123 (2019). https://doi.org/10.1021/acs.jpcb.9b01696.

[55] P. Gabbott, ed., Principles and Applications of Thermal Analysis, Blackwell Publishing Ltd, Oxford, UK, 2008. https://doi.org/10.1002/9780470697702.

[56] J.V.K. L.W.Hill, Dynamic Mechanical and tensile properties in paint and coating testing manual, (2012) 624-636. 